\title{
Measurements in an urban-type boundary layer
}

Received: date / Accepted: date

\begin{abstract}
Wind tunnel measurements of the boundary layer flow over a very rough surface comprising a staggered array of cubes are presented and discussed. Attention is concentrated on the near-wall region, including the canopy region below the tops of the roughness elements. Particle image velocimetry (PIV) and laser Doppler anemometry (LDA) were used to identify the dominant features of the mean and turbulent flow and these are compared with the better-known features of the flow above the roughness. Spatial correlation data, extracted from the PIV images, are used to provide information about eddy structures and it is shown that these differ in some crucial respects from those typical of more classical boundary layers. The implications of the results are discussed in terms of their relevance to flows within the urban environment.
\end{abstract}

Keywords Boundary layer · Roughness · Urban canopies · PIV

Ryan T. Reynolds

School of Engineering Sciences

University of Southampton

Present address:

Lockheed Martin Corporation

E-mail: ryan.reynolds@lmco.com

Ian P. Castro

School of Engineering Sciences

University of Southampton

Highfield

Southampton SO17 1BJ, UK

Tel.: +44-(0)23 80593394

Fax: +44 (0)23 80593058

E-mail: i.castro@soton.ac.uk 


\section{Introduction}

Rough-wall boundary layers have been widely studied, not least because of their relevance to the atmospheric boundary layer which, in conditions of near-neutral stability, is almost invariably aerodynamically fully rough. It has long been thought that provided the roughness height is not too large, its only effect is to change the surface stress, without having any consequences on either the mean flow or the turbulence characteristics throughout the majority of the boundary layer. This is sometimes called Townsend's hypothesis. In a recent review Jiménez (2004) concluded that the extant (laboratory) data is wholly consistent with this view, provided the roughness height $h$ does not exceed some $2-3 \%$ of the boundary layer depth $\delta$. An early example of work which suggests this conclusion is that of Raupach (1981), who showed that neither the inertial layer nor the outer region were modified by roughness. There is, nonetheless, some conflicting evidence that the outer layer turbulence structure can be affected (e.g. Krogstad et al., 1992, for example) even for $h / \delta$ of just a few percent. In any case, there is no doubt that once $h / \delta$ is large enough both the turbulence and the mean flow will have features rather different from normal. This must be true in the region within and just above the roughness, whatever the value of $h / \delta$. One cannot expect Townsend's hypothesis to be appropriate for this roughness sub-layer region, where the flow is strongly inhomogeneous, and it is this region that is the particular focus of the present paper.

In the context of atmospheric flows there is increasing interest in the processes governing pollutant dispersion in the urban environment, for example at street level in a city area. Clearly these processes are dependent largely on the mean and, particularly, the turbulence properties of the flow and these in turn are strongly influenced by the geometric features of the surface. In city-centre areas the average height of the roughness elements (buildings) is often significantly greater than 2-3\% of the boundary layer depth: the latter would normally be in the region of $500 \mathrm{~m}$ in neutral conditions, whereas average building heights could typically be $25 \mathrm{~m}$, with individual buildings significantly taller. But quite apart from issues about the flow above the roughness - like whether a classical log-law region exists even if, at each height, the mean flow is spatially averaged - there is no doubt that the flow beneath the top of the roughness elements will be very different from normal boundary layer flow. There are very few laboratory measurements of the mean and turbulence properties within three-dimensional 'canopy' regions. Some of the first were those of Davidson et al. (1996) and MacDonald (2000) who used pulsed wire anemometry to obtain mean flow profiles at various locations within arrays of cubes. With the assumption of a mixing length constant with height within the canopy, MacDonald (following Cionco, 1972) derived an exponential mean flow profile which agreed tolerably with the spatially-averaged data at least for cube packing densities not too high. Reynolds shear stress measurements were not made, however, so it was not possible to check directly whether the mixing length assumptions were really very consistent with the physics of the canopy flow. 
The precise nature of the flow in the canopy region depends crucially of course on the morphology of the roughness (i.e. the element sizes, shapes and layout). But certain overall features of at least the mean flow have been found to be characteristic of particular classes of morphology - see Raupach et al. (1991), Bottema (1997) and Grachev \& Hunt (2006) for reviews which include some appropriate discussion. Some of the data these authors included are from field campaigns and, more recently, there have been a number of such studies that link particular kinds of flow patterns with diffusional processes within the canopy (see, for example, Rotach et al., 2005, for a field study in Basel, KastnerKlein \& Rotach, 2004, for wind tunnel model results for the city of Nantes, and Poggi et al., 2004b, for the case of a forest canopy). But quite apart from questions concerning pollutant dispersion, there are serious questions concerning how to parameterise the aerodynamic properties of the surface in terms of its morphology. This has been attempted numerous times, most extensively in the context of urban situations by Grimmond \& Oke (1999). Their conclusion was not dissimilar to that of Bottema (1997) who stated that 'problems in obtaining reliable experimental $z_{0}$ and $d$ data (from morphological parameters) are so large that a definitive judgement . . . could not be made'. $z_{0}$ and $d$ are the roughness length and zero-plane displacement that appear in the usual (meteorologists') version of the log-law profile describing the variation of mean streamwise velocity with height,

$$
\frac{U}{u^{*}}=\frac{1}{\kappa} \ln \left(\frac{z-d}{z_{0}}\right)
$$

where $u *$ is the friction velocity defined by $\sqrt{\left(\tau_{w} / \rho\right)}$ with $\tau_{w}$ the wall stress. It is quite clear, however (from Bottema, 1997, amongst a number of others), that for roughness elements of uniform height $(h)$ and all of similar shape, $z_{0} / h$ reaches a maximum value for plan area densities of around $25 \%$. In this paper attention is concentrated not on how to relate aerodynamic parameters to the morphology but rather, for a typical morphology yielding this near-maximum $z_{o} / h$, on the features of the canopy flow and how these are linked (if at all) to those of the flow in that part of the roughness sub-layer just above the canopy. Measurements obtained within the canopy region using laser Doppler anemometry (LDA) and, for the first time, particle image velocimetry (PIV) are presented and their implications discussed. In addition to mean flow data, turbulence stresses and also spatial correlations are presented and the results compared with previous data over similar geometries. Some implications in terms of, inter alia, the adequacy of extant models like that of MacDonald (2000) are discussed. The experimental arrangements are outlined in the following section, with results presented in $\S 3$ and final discussion and conclusions given in $\S 4$. 


\section{Experimental Arrangements}

The experiments were all undertaken in a blow-down wind tunnel whose working section is $4.5 \mathrm{x} 0.9 \mathrm{x}$ $0.6 \mathrm{~m}$, in the School of Engineering Sciences at the University of Southampton. They formed a natural extension to the earlier work reported by Cheng \& Castro (2002) and Castro et al. (2006), hereafter designated $\mathrm{CC}$ and CCR, respectively. The roughness comprised a staggered array of $10 \mathrm{~mm}$ (moulded plastic) cubes covering $25 \%$ of the surface, as illustrated in Fig. 1, and mounted on an 8 mm thick baseboard on the floor of the working section. A leading edge ramp some $200 \mathrm{~mm}$ long was used to sweep the thin contraction-exit boundary layer up to the top of this baseboard. Most data presented here were obtained at a location $3.9 \mathrm{~m}$ from the start of the roughness $(x / h=390)$ and with a free stream velocity, $U_{e}$, of around $10 \mathrm{~m} \mathrm{~s}^{-1}$, giving a momentum thickness Reynolds number of about 13,050. At this location $\delta / h=13.5$ and $u^{*} / U_{e}=0.065$ (see later). Note that even this far downstream the relative roughness height $(h / \delta=0.074)$ significantly exceeds the height generally reckoned to be the limit for unequivocal acceptance of Townsend's hypothesis (see $\S 1$ ).

Measurements of both mean velocity and turbulence stresses were obtained using, firstly, a twocomponent (Dantec Burstware) LDA system operated with an argon-ion laser run at 1 Watt. A fibreoptic probe containing a $300 \mathrm{~mm}$ focal length lens was located outside the tunnel, oriented to yield axial $(x)$ and wall-normal $(z)$ velocity components. This gave a measurement volume $2.39 \mathrm{~mm}$ in length and $0.15 \mathrm{~mm}$ in diameter, some $200 \mathrm{~mm}$ from the spanwise centre-line of the working section. Checks with standard hot wire anemometry showed that this was within the central region where the flow was accurately two-dimensional. (Note that this type of roughness can lead to significant spanwise variabilities near the leading edge, which decay only slowly with downstream distance - see Reynolds et al., 2007.) The Burst Spectrum Analysers were run in coincidence mode and, typically, 100,000 validated bursts were recorded during a sampling time of around 100 seconds. Transit time weighting was used on all statistical averaging to minimise bias errors.

Secondly, two PIV systems were employed. The first used a 1.3 Mpixel HiSense CCD camera with Dantec (Flow Manager 4.1) Burstware and the second, for greater resolution particularly inside and near the canopy region, a 4 Mpixel 12-bit PowerView Plus camera with TSA Insight3G software. In both cases a NewWave Gemini Nd:YAG $120 \mathrm{~mJ}$ laser operating at $532 \mathrm{~nm}$ was used to illuminate the particles. To reduce reflections from the roughness surface, fluorescent FP R6G paint from Flow Visualisations Components was applied to the cube surfaces. This paint absorbs green light (532 $\mathrm{nm})$ and shifts the reflection emission to orange $(625 \mathrm{~nm})$, so the zonal filter at $532 \pm 3 \mathrm{~nm}$ attached to the camera lens allowed only the green light to pass to the camera. Typically, this allowed measurements to within $1 \mathrm{~mm}$ of the cube surfaces, compared to at least $2 \mathrm{~mm}$ without the paint and filter. The image pairs were analysed using adaptive correlation techniques with high-accuracy sub-pixel schemes. Final interrogation windows of either $32 \times 32$ or $16 \times 16$ pixels were used and, when using the high 


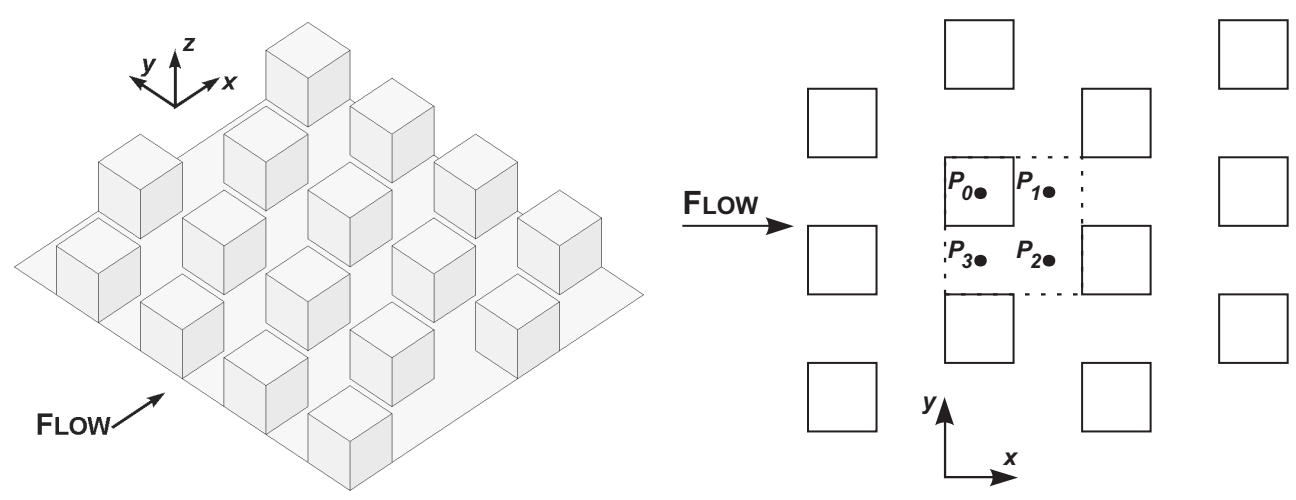

Fig. 1 The roughness array, shown with the flow direction appropriate for the staggered configuration. The four major vertical profile positions, $P_{0}-P_{3}$, are shown at right.

resolution camera, a deformation processing algorithm was also used to increase accuracy further. In this case a $16 \times 16$ pixel interrogation area typically corresponded to a spatial resolution of about $0.4 \times 0.4 \mathrm{~mm}$ and for an $80 \mu \mathrm{s}$ delay between the two laser flashes the dynamic velocity range of the system was about 200 . In all the results presented here vector outliers represented less than $2 \%$ of all vectors, and usually less than $1 \%$; they were removed by appropriate range validation. Code specially developed in LabView (National Instruments) was used to obtain all statistics from the vector maps generated by the Insight3G software, which meant that there were no limits imposed on the number of vector maps that could be processed or the number of vectors per map. 1200 image pairs with $16 \mathrm{x}$ 16 pixel interrogation areas and 50\% overlap on a 2056 x 2056 pixel field of view could be analysed to provide all the statistics (up to second order in turbulence quantities) in about 15 minutes.

Seeding particles for both LDA and PIV measurements were produced by a Safex S 195G smoke generator using Jem Regular DJ Fluid (DJ mix) or Jem Pro Smoke Studio (DX mix) fluids; these are monopropylene glycol in a de-mineralised water suspension and generated particles at the measurement position that were of appropriate size (2-4 microns). In the primary PIV arrangement, the flow was viewed in the $x-z$ plane centred on a streamwise row of cubes (i.e. through the $P_{0}$ and $P_{1}$ positions shown in Fig. 1). The laser was located above the working section and the camera viewed the flow from outside one of the side walls. To maximise resolution the field of view was kept below about $60 \mathrm{~mm}$ square. This meant that four camera positions were needed to cover the entire boundary layer thickness. The lowest position was centred on a cube from the side, so that positions upstream and downstream of the cube could be resolved by looking down the 'streets'. Before presenting detailed results, an example of a time-averaged vector field from this lowest position is shown in Fig. 2. Vectors are coloured with contours of velocity magnitude $\left(\sqrt{U^{2}+W^{2}}\right)$. Usually only a subset of the total derived vectors are shown, for clarity. The actual vector map of Fig. 2b, for example, contained four times as many vectors as shown. A $50 \%$ overlap of interrogation windows was used because this minimises errors arising in 

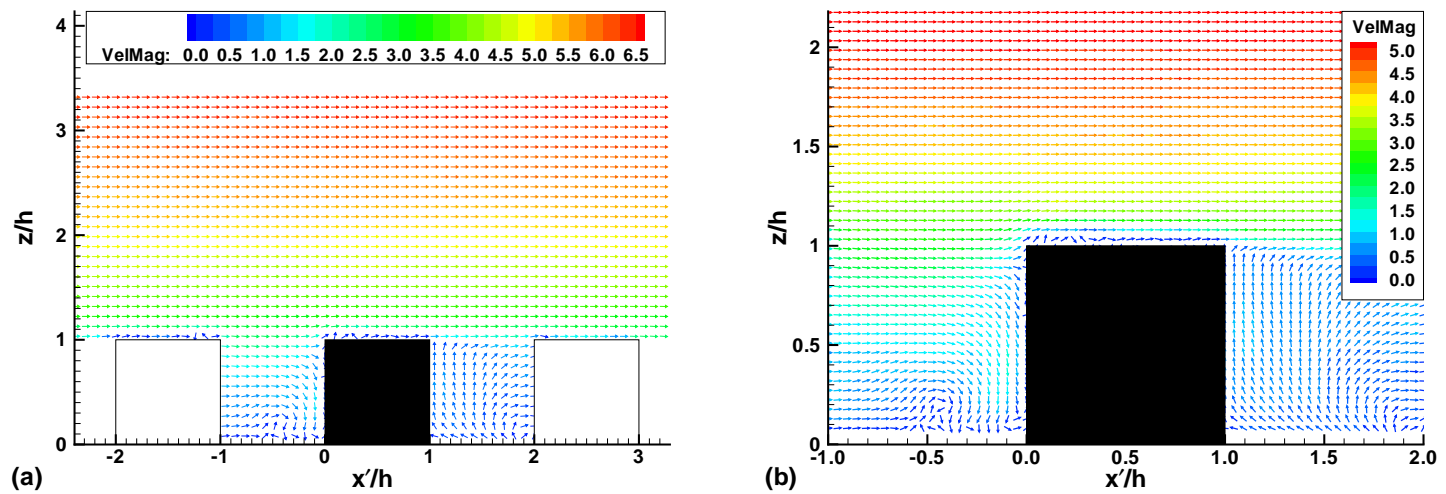

Fig. 2 Mean velocity vector maps at the lowest camera position. Flow is from left to right. The lower portion of the image is below the floor and thus unresolved and the white cubes are out of the plane of the laser sheet. The right-hand image is a magnified version of the area around the central cube. Arrow lengths are constant, with velocity magnitude indicated by the colour scale.

the algorithm used to replace the very small number of 'bad' vectors, but it does not of course increase the inherent spatial resolution. Magnified versions of these images will be presented and discussed in due course but notice the small recirculating region visible just upstream of the central cube and the much larger one downstream.

Some additional measurements were made using standard crossed-hot-wire anemometry (HWA). The probes had wire angles of $\pm 60^{0}$ (unlike the standard $\pm 45^{0}$ ) to minimise errors in the high turbulence region in the roughness sub-layer. They were driven by University of Newcastle (NSW) bridges whose outputs were filtered and massaged by appropriate gain and offset to allow best use of the analogue-digital convertors (IOTech ADC488). Calibrations, using the effective cosine law for yaw calibration (typically over yaw angles up to $\pm 40^{\circ}$ ), were performed in the free stream against a standard Pitot-static tube using a Furness micro-manometer whose output was passed to the same A/D system. Specialised software written in LabVIEW allowed on-line calibration and measurement of all necessary quantities. Sampling rates were typically between 2 and $10 \mathrm{kHz}$, with sampling times of 60-120 s.

\section{Results}

In the following sections we present and discuss all the major results. Although the specific emphasis is on the nature of the flow within the canopy region we start, in $\S 3.1$, by presenting details of the complete boundary layer. The data here largely confirm that the roughness does not significantly affect mean velocity and stress profiles in the outer region of the flow, which is in accordance with conventional wisdom (although recall that at the measurement location $h / \delta$ is quite high -0.074 ). $\S 3.2$ discusses the nature of the mean flow and turbulence Reynolds stresses in the canopy region. 

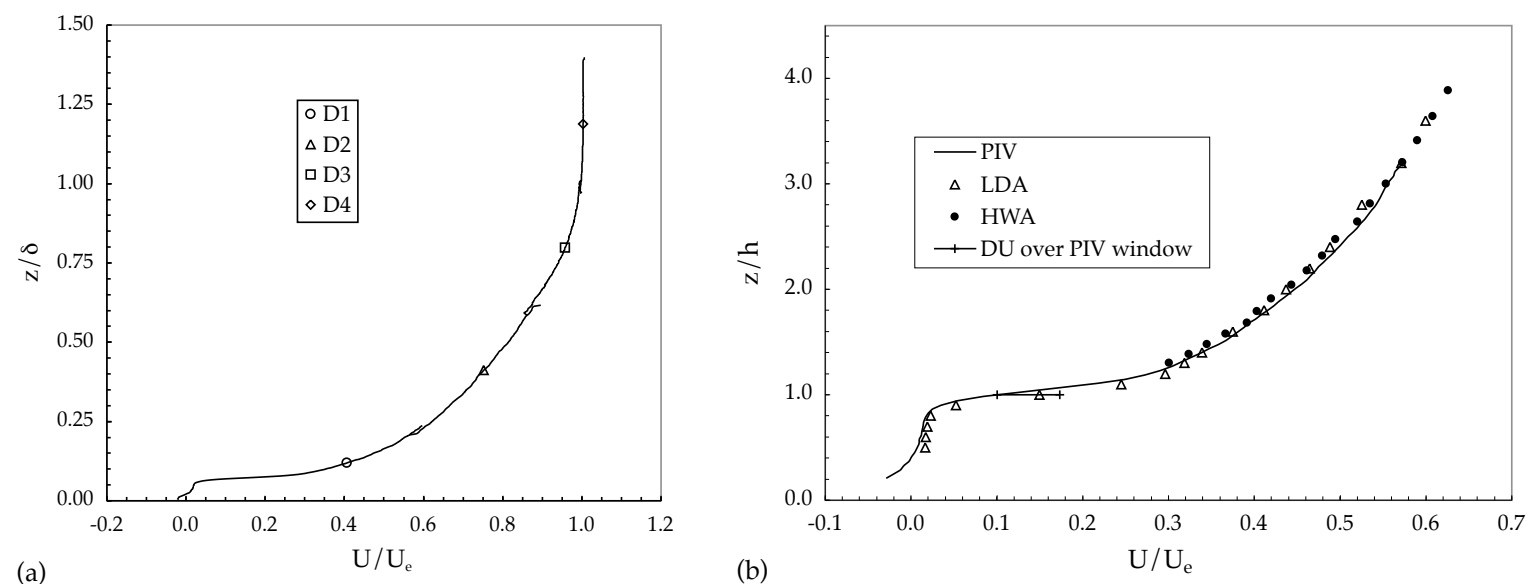

Fig. 3 Mean velocity profiles at $P_{1}$ : (a) across the entire boundary layer, from four PIV images; (b) in the near-surface region, from PIV, LDA and HWA.

The flow is compared and contrasted with that over an isolated cube within a thick boundary layer there are naturally some similarities but also some significant differences. Initial conclusions about the turbulence structure are made, principally that the turbulence is on the whole much more isotropic in terms of the stress distributions than it is in smooth-wall flows. In $\S 3.3$ the turbulence structure is explored in more detail using quadrant analysis and consideration of integral scale data (which can be obtained directly from PIV data). Amongst other things, the results suggest a flow which has at least some similarities with those found in dense vegetation canopies.

\subsection{Overall boundary layer details}

Fig. 3a shows mean axial velocity profiles obtained at a location just behind a cube $\left(P_{1}\right.$, see Fig. 1$)$, extracted from the time-averaged PIV vector maps (like those in Fig. 2) at four camera positions (D1D4). The data were obtained from $0.4 \times 0.4 \mathrm{~mm}(16 \times 16$ pixel $)$ interrogation areas and are represented by lines drawn through each point with, for clarity, the points not shown individually. Each individual point is an average over three consecutive interrogation areas in the axial direction. A single point from the middle of each domain is shown and the minimal mismatch in the overlap regions can just be discerned. Some of this is a result of rather greater errors from interrogation areas on the image domain boundaries. Fig. 3b shows similar data in the region below $z / h=4$ (the D1 domain of Fig. 3a) compared with data obtained from an LDA traverse and a similar HWA traverse (in the region where the latter was viable because the turbulence intensities were not too high). Agreement is very satisfactory, although careful scrutiny of the data near the cube top, where the mean shear is at its greatest, suggests that the PIV technique, obtained in this case with a $0.8 \times 0.8 \mathrm{~mm}(32 \times 32$ pixel $)$ interrogation window size, yields noticeably lower values of mean velocity than given by the LDA, 

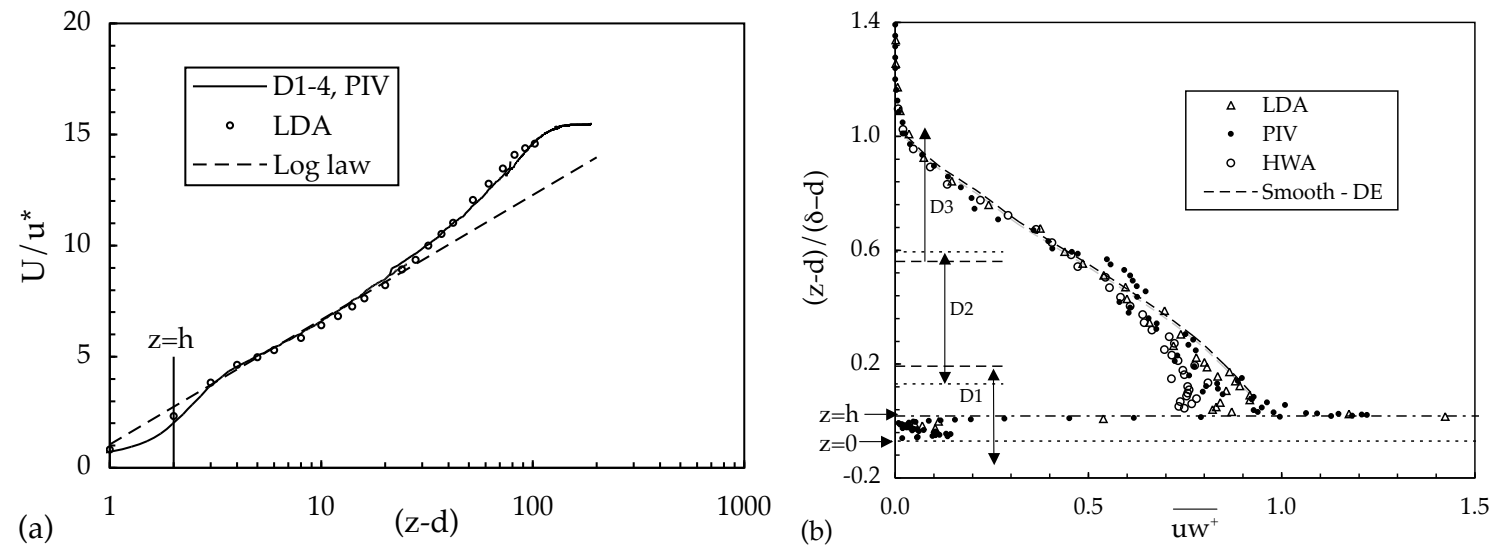

Fig. 4 (a): Mean velocity profile at $P_{1}$ in log-law form. (b): Reynolds shear stress profile at $P_{1}$; the vertical extent of the PIV image domains D1-D3 are indicated.

\begin{tabular}{cccccccc}
\hline$x$ & $\approx U_{e}$ & $x / h$ & $\delta / h$ & $u^{*} / U_{e}$ & $d / h$ & $z_{o} / h$ & $R e_{\theta}$ \\
$\mathrm{m}$ & $\mathrm{m} \mathrm{s}^{-1}$ & & & & & & \\
3.9 & 10.8 & 390 & 13.5 & 0.065 & 0.65 & 0.065 & 13,050
\end{tabular}

Table 1 Salient boundary layer parameters. $x$ measured from the leading edge of the roughness.

which has a much smaller spatial resolution (measurement volume diameter about $150 \mu m$ ). This is consistent with the effects of a significant shear over the interrogation window size; the change in mean velocity over a $0.8 \mathrm{~mm}$ change in $z$ in this high-shear region is indicated Fig. 3b and it was this resolution issue that led to all subsequent measurements (including those in Fig. 3a) being made with the higher resolution system.

The major boundary layer parameters at the axial measurement station are summarised in Table 1 and mean velocity data are shown plotted in log-linear form, equation (1), in Fig. 4a. CC have demonstrated that the average turbulence shear stress in the log-law (inertial sub-layer) region, $-\rho \overline{u w}_{i}$ say, does not provide an accurate estimate of the wall stress for this type of boundary layer. They showed via measurements of surface drag using pressure-tapped cubes that $-\rho \overline{u w}_{i}$ underestimates the surface stress by some $24 \%$. So in the present case the friction velocity was estimated by taking $u^{*} / U_{e}=1.12 \sqrt{-\overline{u w_{i}}}$, with the inertial sublayer taken as the region defined by $0.14<z / \delta<0.22$ $(2<z / h<3)$. Fig. $4 \mathrm{~b}$ shows shear stress profile data at $P_{1}$ from PIV, LDA and HWA and within the expected accuracy of all three techniques the data collapse reasonably well, although it is noticeable that in the near-wall region the HWA data are somewhat lower than those obtained by LDA or PIV, despite the use of cross-wire probes of unusually high included angle (see §2). It seems likely that even these high angles and the correction routines used (based on the data of Tutu \& Chevray, 1978) are insufficient to remove all the inadequacies of cross-wire anemometry in such high turbulence regions. HWA data were not of course obtained below about $z / h=1.2$. Within the inertial sublayer the average 

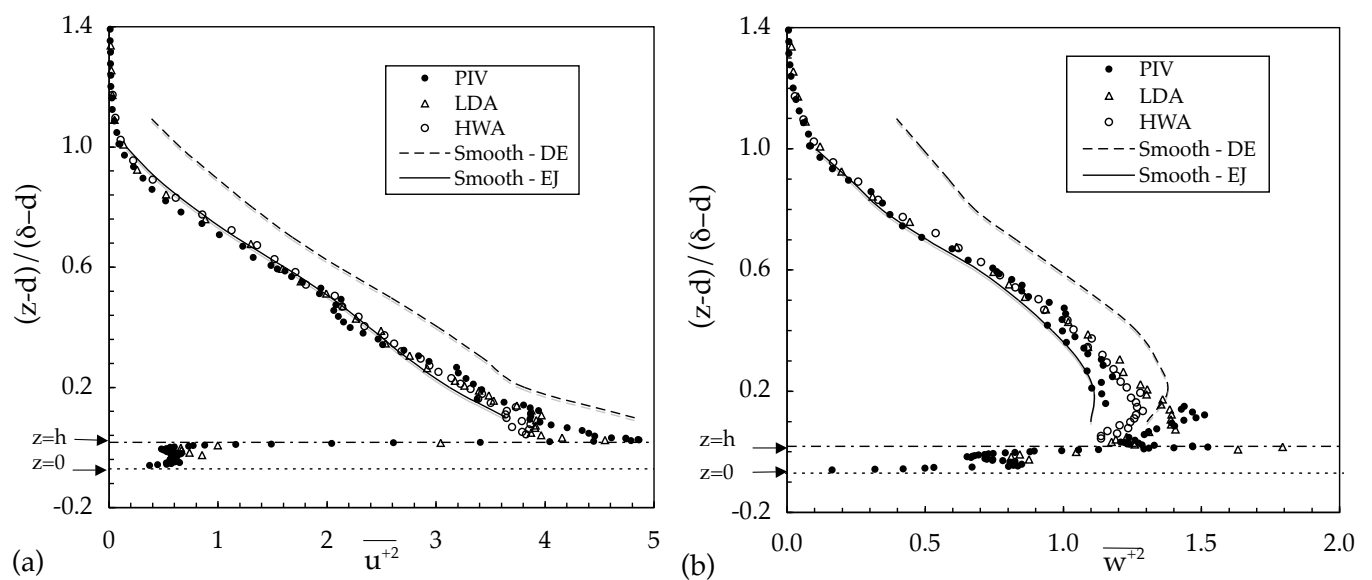

Fig. 5 Reynolds normal stress profiles at $P_{1}$. (a): $\overline{u^{2}} / u^{* 2} ;(\mathrm{b}): \overline{w^{2}} / u^{* 2}$.

normalised shear stress is about 0.0034 , so that $u^{*} / U_{e}=0.065$. The mean velocity data shown in Fig. 4a have a slope clearly consistent with this value (using a zero-plane displacement of $0.8 h$ ) and yield $z_{0} / h=0.065$, similar to the value found at this fetch by CC. Note that the data depart from the log-law in the region just above the cube top; this is not surprising because the flow remains spatially inhomogeneous in horizontal planes for $z / h \leq 1.8$ (see CC for a full discussion).

Reynolds normal stresses are shown in Fig. 5. To reduce scatter, above about $z / \delta=0.1$ each PIV data point shown in the figures is an average of those in an area of $3 \times 5$ interrogation windows (i.e. an area of $1.2 \times 2 \mathrm{~mm}$ ). The scatter in the PIV normal stress data is noticeably lower than for the shear stress data, which is perhaps not surprising - the latter provide a particularly stringent test of PIV accuracy when making measurements in air and recall that only 1200 image pairs were used to obtain the statistics. Nonetheless, the general agreement between the data obtained using the different techniques is again very satisfying. The data in both Fig.4b and Fig.5 are plotted using a wall distance that takes account of the zero-plane displacement $(d)$. Whilst it is not our purpose in this paper to discuss in detail the overall stress profiles compared with corresponding smooth wall profiles, this allows such a comparison and the figures include data from Erm \& Joubert (1991) and DeGraaff \& Eaton (2000) as examples of the latter. Some comments are appropriate.

Erm \& Joubert's data had $R e_{\theta}=2788$ and the DeGraaff \& Eaton results shown in Fig. 5 are represented by smooth lines through their data for $R e_{\theta}=13,000$, which is very close to the present value $(13,050)$. DeGraaff \& Eaton found some evidence of $R e$ effects on $\overline{u^{+2}}$, with the data at $R e_{\theta}=$ 2,900 somewhat lower than at higher $R e_{\theta}$. Variations in $\overline{w^{+2}}$ were rather smaller but, in any case, their data at all $R e_{\theta}$ are significantly higher than Erm \& Joubert's. They also seem unrealistically high near the edge of the boundary layer, which cannot be explained by the (very small) differences in measured $u^{*}$ in the two experiments. For the present rough-wall experiments it should be emphasised 
that the profiles are largely independent of $R e_{\theta}$ even in the near-wall region, as the surface drag is almost entirely form drag. If one accepts the Erm \& Joubert data as typical of smooth-wall results, it would seem that even the present degree of roughness does not lead to significant Reynolds stress differences in the outer layer, provided the data are normalised by $u^{* 2}$. This is in contrast to the conclusion of Krogstad et al (1992) who found that over a mesh-type roughness, whilst there was only a marginal increase in $\overline{u^{+2}}$ compared with their smooth wall results (which were also close to the Erm \& Joubert data shown here), there was a significant increase in $\overline{w^{+2}}$, which reached values around 1.8 near $z / \delta=0.1$. The reason for this difference between the two surfaces is currently unclear and warrants further study. What is clear, however, is that in the present case, despite $h / \delta$ being as high as 0.074 (significantly larger than the 2-3\% that Jiménez, 2000, suggested would be an upper limit for adequacy of the Townsend hypothesis), the smooth and rough wall normalised stress profiles collapse throughout the outer part of the flow.

It is worth noting that the mechanics of the flow above the roughness could in principle be discussed in the light of'blocking' ideas on turbulence near interfacial layers, as recently explored in detail by Hunt et al. (2006), but that is not pursued here. For the present purposes the point to emphasise is the general level of agreement in the stresses obtained by the three different instrumentation techniques. There are, however, significant differences within the region around the top of the roughness elements. This is a result of the different spatial resolution for the LDA and PIV systems, discussed earlier in the context of the mean velocity data (Fig. 3b). In this region (below $z / h=2$, say) both the mean flow and the turbulence stresses also depend of course on the specific profile location; this is explored further in 3.2 .

\subsection{Basic near-wall \&3 canopy flow details}

We begin by considering briefly the mean velocity field. The near-wall spatial inhomogeneity generated by the roughness is illustrated in Fig. 6, which shows mean velocity vectors just ahead of and behind a cube, on its central plane. These are enlarged versions of sections from Fig. 2. The data shown in Fig. 6b show a flow topology upstream of the cube face not unlike that for boundary layer flow over an isolated cube. Although data are not available nearer than about $1 \mathrm{~mm}$ from the surface, the identified vectors suggest a separation saddle point at a distance around $-0.55 h$ from the upstream face, marking the upstream limit of the well-known horseshoe vortex that curls spanwise around the cube. There is (perhaps) a subsequent attachment saddle point near $-0.3 h$. The latter is fed by flow from above which is separated from the flow over the cube by an attachment on the front face near the top - around $z / h=0.9$. Precise details cannot be determined unequivocally, but there are clearly great similarities with the topologies identified by, for example, Castro \& Robins (1977) for an isolated cube in a thick boundary layer and Martinuzzi \& Tropea (1993) for the corresponding channel flow. 

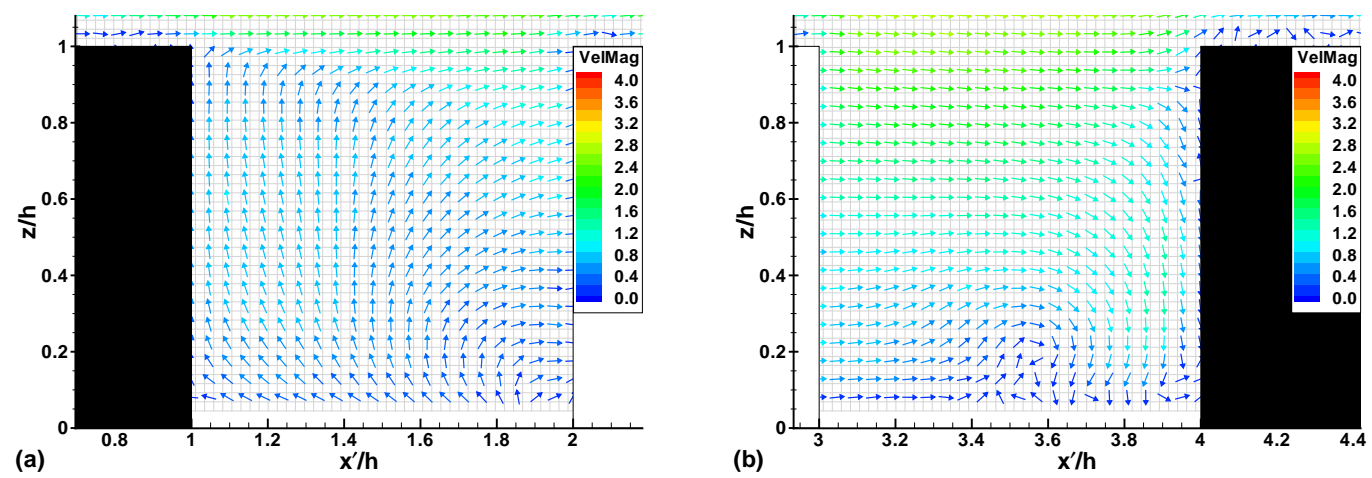

Fig. 6 PIV Mean velocity vectors on the vertical plane through the centre of the cubes, downstream (a) and upstream (b) of a cube. The outer boundary layer flow is from left to right, with $x^{\prime}=0$ located at the front face of the upstream cube.

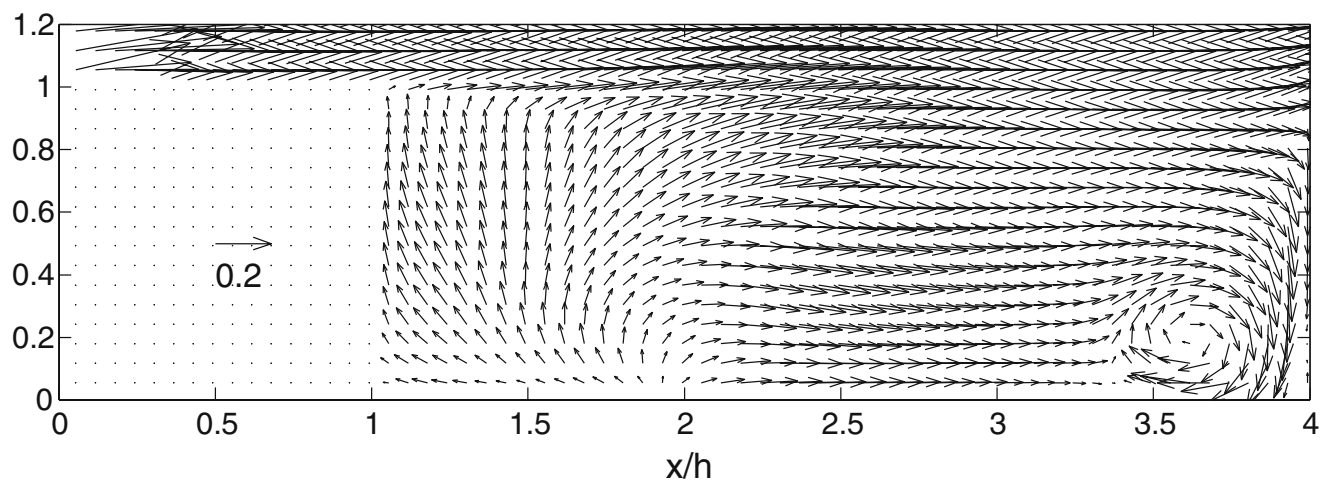

Fig. 7 Mean velocity vectors between two cubes, on a vertical plane through the centre of the cubes. From the DNS computations of Coceal et al (2006). Flow from left to right, with $x=0$ located at the front face of the upstream cube.

The flow downstream of each cube is, however, very different from that for an isolated cube flow, where there is generally a clear attachment region on the surface in the near wake. Fig. 6a shows, in distinct contrast, a separation saddle on the surface near a location $0.9 h$ downstream of the rear face. Near the $x=0$ plane the flow must sweep in towards the centre plane $(y=0)$ from the sides; the shear layer near $z=h$, rather than being swept downwards to the surface, remains high in the canopy, attaching on the upstream face of the downstream cube as indicated above. These data are entirely consistent with the results of a well-resolved DNS calculation of channel flow over an identical cube array (Coceal et al, 2006). Vectors from this calculation are shown in Fig. 7 and the agreement is clear.

It is worth pointing out that because of the strong Reynolds stress gradients the link between the mean pressure field and the mean velocity field within the canopy region is much weaker than it is in other kinds of flows - especially those governed essentially by inviscid dynamics. So, for example, whilst on the front face the measurements of $\mathrm{CC}$ showed that the surface pressure is a maximum around the 

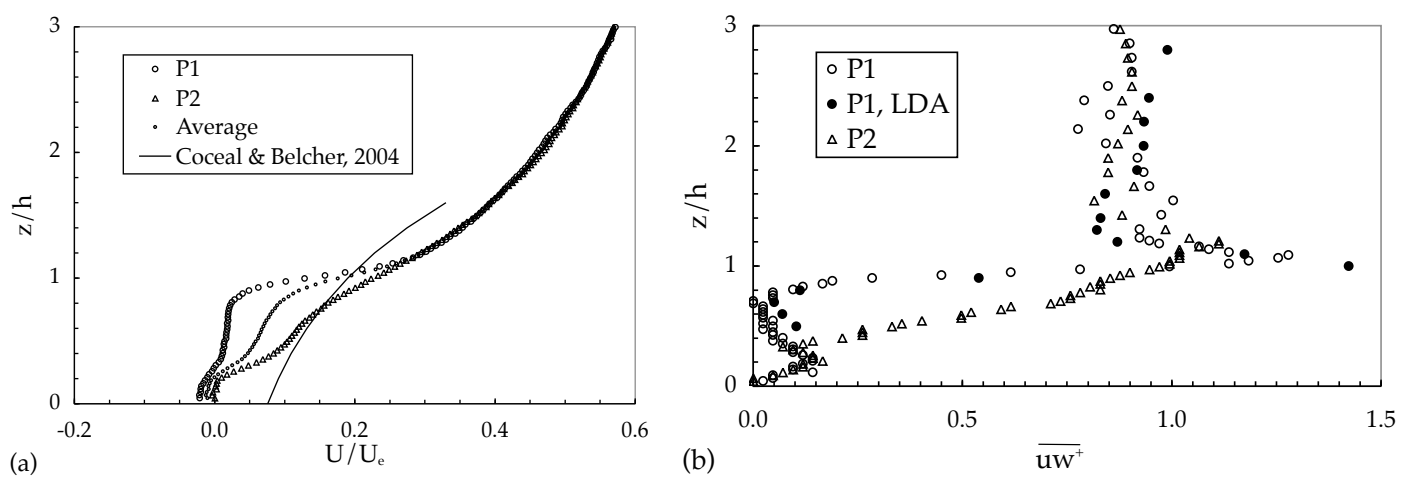

Fig. 8 Mean velocity and shear stress profiles at two locations in the canopy region.

attachment point (near $z / h=0.9$ ) and falls to a minimum around $z / h=0.25$, with the near-surface flow moving downwards as might thus be expected, there is an equally clear flow up the rear face of each cube (see Fig. 7) even though the rear-surface pressure is closely uniform, as indeed it is for flow over an isolated cube.

An obvious feature of the mean flow is the strong shear region around the top of the canopy, all the way from one cube to the next, but detailed individual vertical profiles depend strongly on spatial location, as discussed in CC (and Coceal et al, 2006) and illustrated in Figs. 8 and 9 using the present PIV and (for P1) LDA data. Profiles at P1 and P2, i.e. behind and ahead of the cube, are shown. These are very similar to those of CC, but not identical since the relative boundary layer thickness was significantly higher in this latter case. Fig. 8a includes the profile resulting from spatial averaging of numerous profiles obtained within the 'repeating unit' area (see CC for details) and it is clearly not dissimilar from the average of the two profiles shown - which are thus in some sense representative. There have been a number of models suggested for the spatially averaged mean flow profile within the canopy. With $U_{h}$ defined as the average velocity at the top of the roughness MacDonald (2000) proposed the form

$$
U=U_{h} e^{a(z / h-1)}
$$

where $a$ is a constant which, for the present surface, is about 2.4. An alternative and arguably more soundly-based version of this was suggested by Coceal \& Belcher (2004), who used a quite different morphometric approach coupled with a mixing length model to derive the coefficient. They suggested

$$
U=U_{h} e^{(z-h) / l_{s}}
$$

with, for this case, $l_{s} / h=1.07$ (equivalent to $a=0.93$ ). This profile is included in Fig. 8a.

The major limitation of these models is that they do not contain the inflection point in the shear layer region around the top of the roughness which is a ubiquitous feature of the measured profiles. 

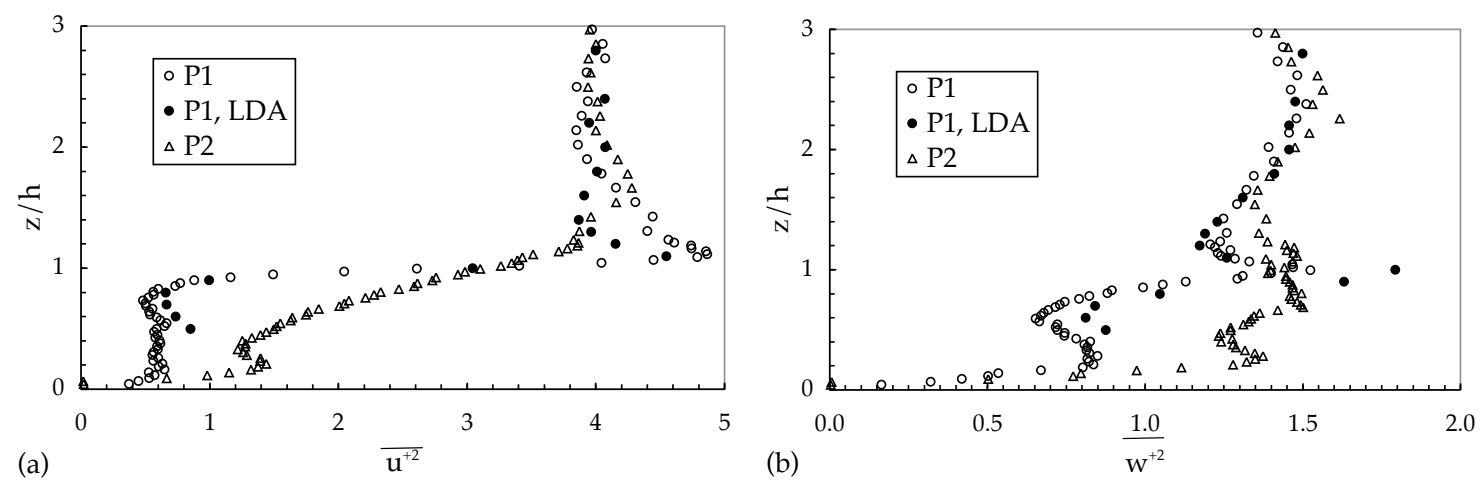

Fig. 9 Reynolds normal stress profiles at two locations in the canopy region.

Indeed, these inflection points provide a means for rather different instability mechanisms than those which occur in smooth-wall flows. This has been widely discussed in the context of plant canopies and Finnigan (2000) has given a thorough review of the topic. Nearer the base of the canopy, the variations in shear stress follow those in the gradient of the mean velocity. So, for example, where the mean flow gradient is close to zero $(0.4<z / h<0.8$ for P1, Fig. 8a) then so is the shear stress (Fig. 8b). Similarly, even nearer the surface where the mean flow gradient rises again (marking the edge of the separated zone at this axial location), the shear stress also rises to another small peak.

The shear layer near the canopy top is characterised by significant peaks in the Reynolds stresses, evident in Figs. 8b and 9. As noted earlier, there are resolution issues in this strongly sheared region so that the PIV data show rather lower values of the peak stresses $\left(\overline{u w^{+}}\right.$and $\overline{w^{+2}}$ ) than those given by the LDA. Measurements at different axial locations showed that the peak values are dependent on $\delta / h$, as shown in Fig. 10. Increasing $\delta / h$ corresponds to increasing $\theta / z_{0}$ since $z_{0}$ is, like $h$, constant with fetch $(x)$. There is clearly a monotonic fall in the peak stresses (Fig. 10a), but some of this is due to the inevitable fall in wall stress with increasing $\theta / z_{0}$ (corresponding to $\operatorname{Re}_{\theta}$ in a smooth-wall boundary layer, see Castro, 2007). Normalising by $u^{* 2}$ as in Fig. 10b suggests that the peak stresses asymptote to fixed values, as might be expected for a developed boundary layer. Note, however, that $h / \delta$ certainly needs to be below $10 \%$ and perhaps even below $5 \%$ before this asymptote is reached.

The turbulence structure within classical mixing layers, let alone the complex canopy flow, is very different from that in the near-wall region of boundary layers, so turbulence parameters like $-\overline{u w} / u^{\prime} w^{\prime}$ and $\overline{w^{2}} / \overline{u^{2}}$ vary widely in the canopy region; (here $u^{\prime}$ and $w^{\prime}$ refer to rms values of the fluctuating velocities). This is illustrated in Fig. 11, which shows these ratios at P1 and P2. Values of both parameters lie between about 0.3 and 0.4 in the near-wall region of smooth-wall boundary layers, which is indicated on the figure. What might seem particularly significant, however, is that $\overline{w^{2}} / \overline{u^{2}}$ can actually exceed unity by a considerable margin in some regions. Near $z / h=0.82$ for example, which is a little below the centre of the shear layer if the latter is defined as being where the peak stresses 

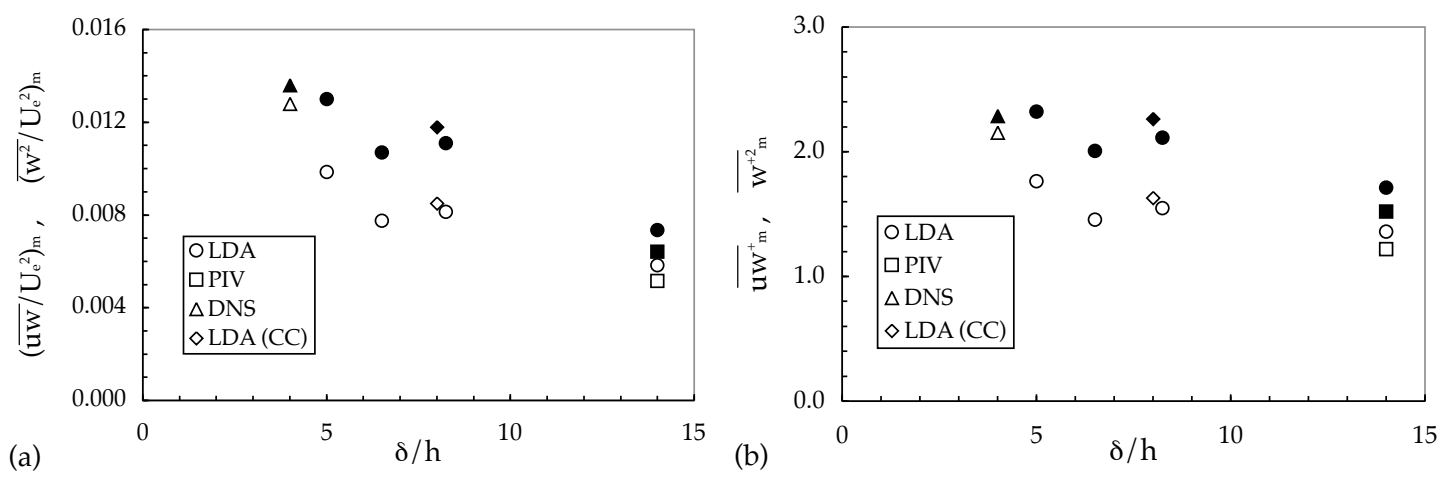

Fig. 10 Peak Reynolds shear and vertical normal stresses at P1 as a function of axial position. (a): normalised by (a) $U_{e}^{2}$ and (b) $u^{* 2}$. Solid symbols refer to $\overline{w_{m}^{2}}$.

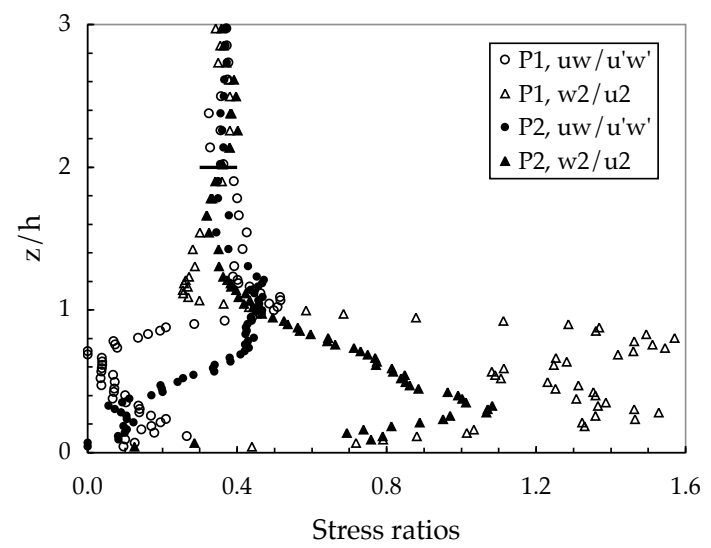

Fig. 11 Structure parameters in the wall-region. Values for both parameters within smooth-wall boundary layers are indicated by the solid horizontal line at $z / h=2$. Open and solid symbols refer to $\mathrm{P} 1$ and $\mathrm{P} 2$ respectively. Circles: $\overline{u w} / u^{\prime} w^{\prime}$; triangles: $\overline{w^{2}} / \overline{u^{2}}$.

occur, $\overline{w^{2}} / \overline{u^{2}}=1.5$. A similar value is reached around $z / h=0.4$. This value is very much higher than the peak $\overline{w^{2}} / \overline{u^{2}}$ (typically around 0.5 ) that occurs in classical plane mixing layers. However, this is almost certainly a result of the shift in the mean flow direction. In fact, Fig. 6a or Fig. 7 indicate that at the $\mathrm{P} 1$ location $-0.5 h$ from the rear face - the average flow direction is actually nearly vertical. Individual stress ratios depend strongly on the coordinate system, of course, and strictly should be referred to a system aligned with the mean flow. At $z / h=0.8$, with respect to the local flow direction the appropriate ratio of cross-stream to axial stresses is thus more nearly $\overline{u^{2}} / \overline{w^{2}}$, which is (at about 0.67) rather closer to classical shear flow values. Using an analysis of the principle invariants of the anisotropy tensor $\left(b_{i j}=\overline{u_{i} u_{j}} / \overline{q^{2}}-\delta_{i j} / 3\right)$ CCR have shown that deep within the canopy region - at least at P1 and P2 - the turbulence is in fact much more closely isotropic than it is in regular shear flows of any kind. 

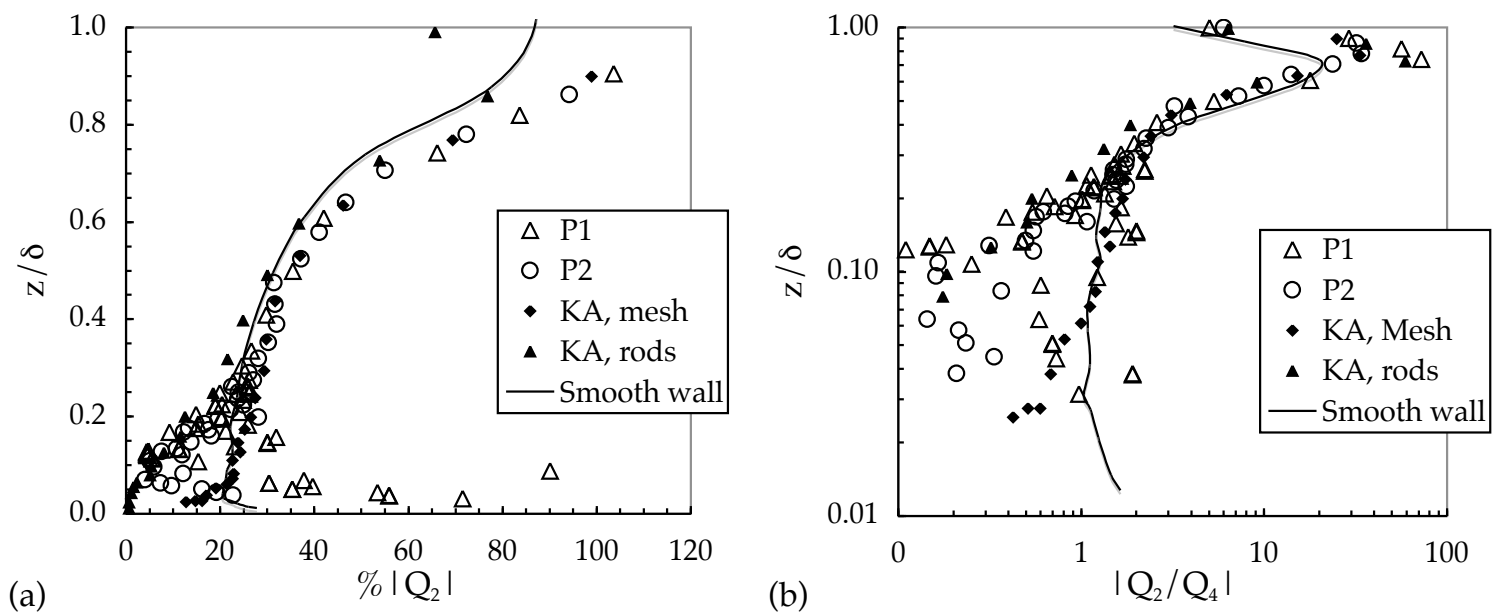

Fig. 12 (a): percentage contributions to $-\overline{u w}$ from quadrant 2 (ejections); (b); ratio of ejections to sweeps. $H=2.5$. The mesh and $(2 \mathrm{D})$ rod roughness data and the smooth wall data are from Krogstad \& Antonia (1999).

\subsection{Turbulence structure measurements}

Lu \& Willmarth (1973) were perhaps the first to quantify the proportion of shear stress generated by motions in the different quadrants of the $u-w($ or $u-v$ ) plane. CCR showed, using that technique, that within the roughness sublayer (above the canopy region) 'sweep' events, for which $u>0, w<0$, are significantly more dominant than above smooth walls. Furthermore, the events that contribute most to the momentum transfer are those having largest amplitudes. The contribution to $\overline{u w}$ from a particular quadrant can be written as

$$
\overline{(u w)}_{Q}=\lim _{T \rightarrow \infty} \frac{1}{T} \int_{0}^{T} u w I_{Q}(t) d t
$$

where $I_{Q}(t)$ is defined to be unity if $|u w|=H u^{\prime} w^{\prime}$ and zero otherwise. $\left(u^{\prime}\right.$ and $w^{\prime}$ denote the rms values of the two components of the fluctuating velocities). Choosing the threshold level as $H=2.5$, for example, excludes contributions arising from relatively low values of the velocity products $-u w<6 \overline{u w}$ - and thus emphasises the contributions from the largest amplitude events. Fig. 12 shows examples of percentages of the total shear stress provided by such events, for quadrant two (ejections), and the ratio of contributions from quadrants two and four (i.e. ejections/sweeps). It is clear that in the outer region of the flow ejections dominate and the results are largely independent of the nature of the rough surface. The slightly lower value of $\left|Q_{2}\right|$ at a given $z / \delta$ for the smooth surface may be a genuine affect of roughness but it could also be a Reynolds number effect; quadrant analysis data in smooth-wall boundary layers are known to be somewhat Reynolds-number dependent. Data from Krogstad \& Antonia (1999) for a three-dimensional mesh-type roughness, two-dimensional spanwise 

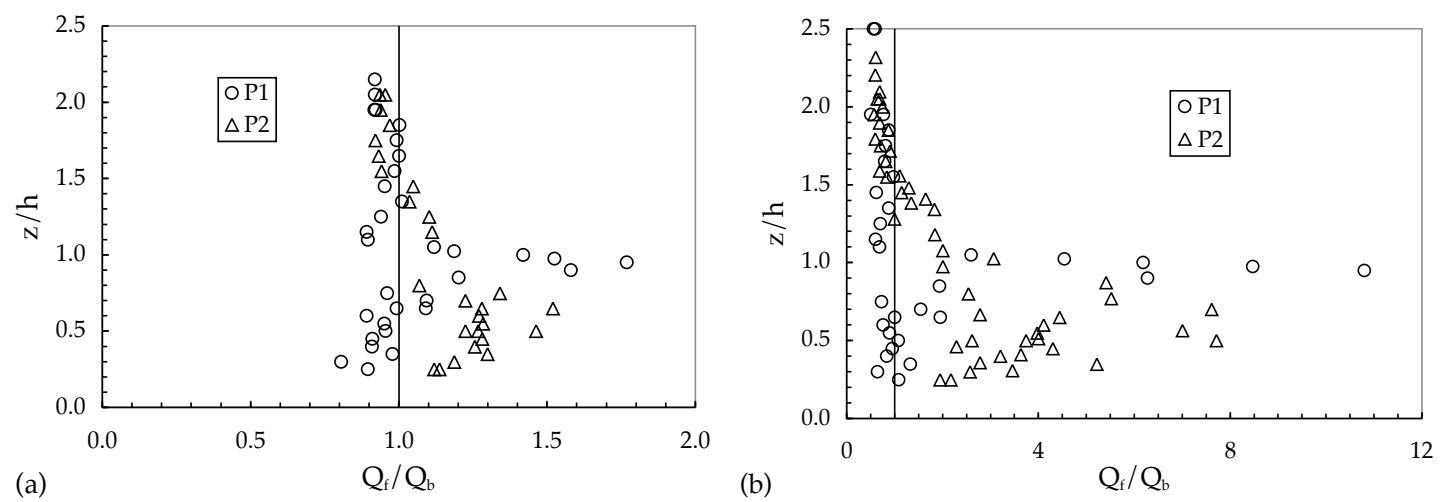

Fig. 13 (a): Ratio of the proportions of forward motions to backward motions in the $x-z$ plane for (a), $H=0$ and (b), $H=2.5$

bars and smooth-wall conditions are included in the figure. Only within the roughness sublayer do the different surfaces lead to significant variations in behaviour. Within the canopy region (i.e. below about $z / \delta=0.07$ for the present surface - P1 and P2 data in fig.12b) sweeps are very dominant at P2, but less so at P1, where the mean flow is often nearly vertical. Note that the Krogstad \& Antonia data do not extend below $z=h$, so their results for the mesh surface at the lowest $z / \delta$ values in fig.12b are actually within the roughness sublayer above the canopy itself.

Fig. 13 emphasises the importance of forward-biased motions for the roughness sublayer region. These are dominated by sweeps as quadrant 1 and 3 events are relatively weak, independent of $H$, as commonly found in wall turbulence. Note that, even without any weighting towards the largest amplitude motions, forward motions are dominant around the top of the canopy and, at P2, well within the canopy. The effect is much stronger with $H=2.5$ (Fig. 13b); scatter in this figure is noticeably greater because there were far fewer occurrences of the more extreme velocity pairs in the quadrants than when $H=0$, but the trend is very clear. Time records of simultaneous $U$ and $V$ velocities were also available - from LDA data obtained with the beams entering the canopy from below rather than the side. Quadrant analysis of these data gave results consistent with the data in Fig. 13 in that forward motions were equally dominant in the region around $z=h$. The prevailing sweep motions $(u>0, w<0)$ can be identified in snapshots of the fluctuating velocity vectors from the PIV data. Fig. 14 shows two such snapshots. In Fig. 14a, in which one such event is indicated, strong positive fluctuations (coloured red) are clearly seen in the region just above the canopy, where the mean flow shear is greatest. In both figures such sweeps can be identified elsewhere within the domain, but on average the quantitative data, as noted above, indicate their dominance in the region around $z=h$. 

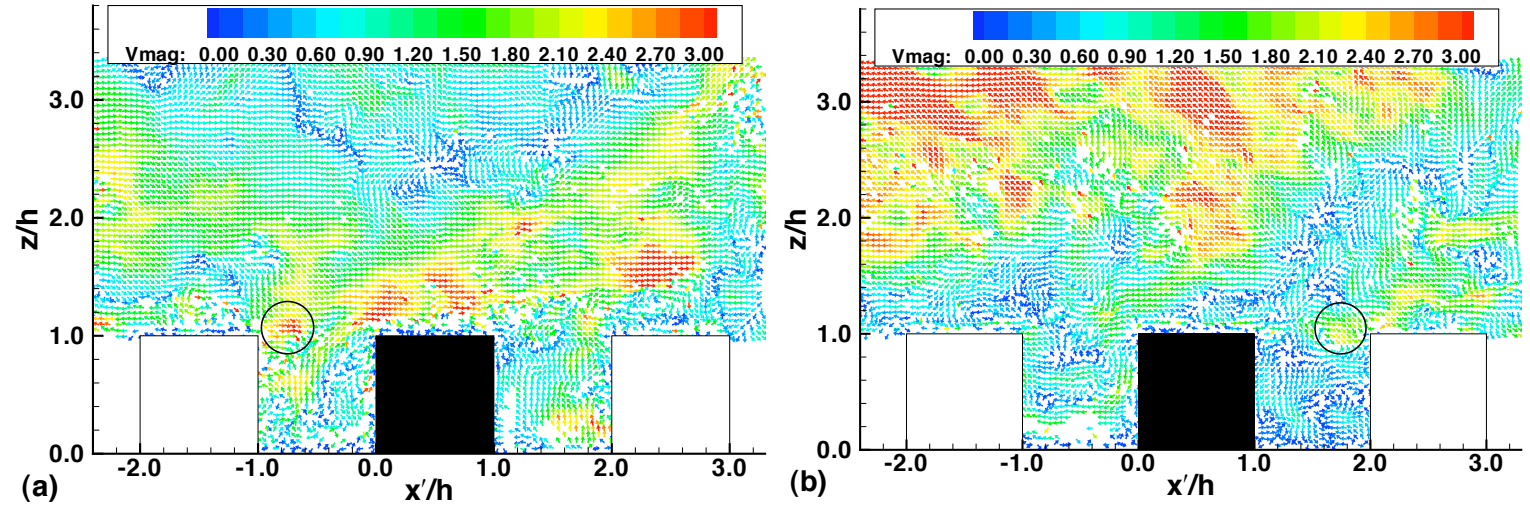

Fig. 14 PIV instantaneous mean-subtracted velocity vectors around the cubes, on the central plane over the central (black) cube. Flow is from left to right. Circles enclose a noticeable sweep event.

The results discussed above are quite similar, qualitatively at least, to those found for boundary layers above vegetation canopies, despite the very different nature of the inhomogeneities of the canopy geometry. Poggi et al. (2004b), for example, found that the region above such a canopy is dominated by ejections whereas the momentum flux within the canopy is controlled largely by strong sweeps provided the canopy is sufficiently dense. So it appears that in this respect dense vegetation canopies contain flows which can be similar to those within urban (building-type) canopies, as will be noted again below.

The availability of PIV data allows more extensive extraction of two-point spatial correlations than is practical using two individual probes. The latter technique was employed by CCR for the same roughness geometry and it was shown that the structure angle $\theta$ - the most likely inclination of the 'average' eddy structure - decreases with height in the region above the canopy. This trend is opposite to that found in smooth-wall flows (Adrian et al., 2000, Marusic, 2001). Fig. 15a shows contours of the two point spatial correlation of the axial fluctuating velocity, obtained for a fixed 'probe' (reference) position at $z / h=1.5$ above the centre of a cube, derived from 1200 PIV snapshots. With such a limited set of data there is some inevitable 'fuzziness' in the contours, but the figure clearly shows a measurable inclination of the average structure at that location. For this same P0 location, similar contour plots were derived at various points and the resulting variation in structure angle with height is shown in Fig. 15b. The data agree well with those of CCR, obtained using a totally different technique - time-delayed spatial correlations from simultaneous cross-wire and LDA signals at two points. They demonstrate a significant rise in $\theta$ with decreasing $z$ through the roughness sub-layer, which is largely independent of $h / \delta$ even though the latter differs by almost a factor of two between the two experiments. In contrast, the smooth-wall data used by Marusic (2001) to compare with theoretical eddy structure models show $\theta$ increasing monotonically from about $10^{\circ}$ near the wall to nearer $35^{\circ}$ at $z / \delta=0.22$, which in the present terms corresponds to about $z / h=3.0$. This is clearly very different to the trend 

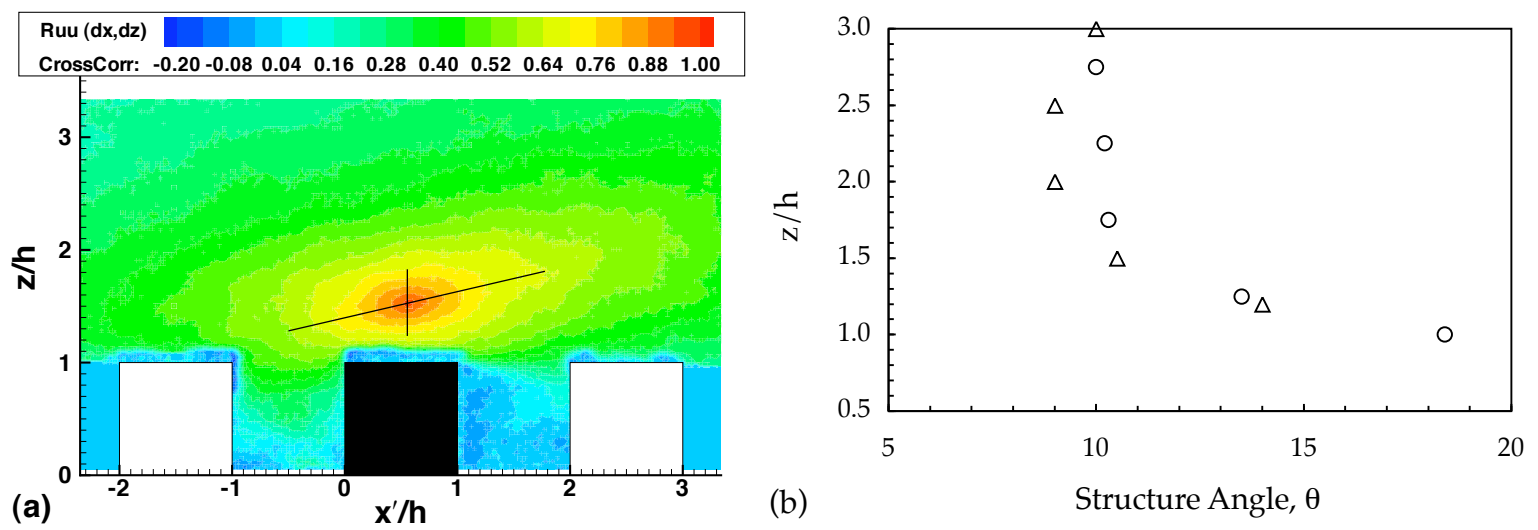

Fig. 15 (a): contours of constant spatial correlation, $R_{u u}$, for $z$ near $1.5 h$ over the centre of the cube, from PIV data. (b): structure angle, $\theta$, deduced from figures like (a), as a function of $z / h$, the fixed point location. Triangles refer to PIV data $(\delta / h=14)$, circles to CC's two-probe data $(\delta / h=8)$.

indicated by the data in Fig. 15b. One might expect the data at larger $z / h$ to increase again, becoming similar to those in smooth-wall flows; this point was not pursued but the rather different nature of the turbulence structures in the roughness sublayer from those in the surface layer above a smooth-wall is quite evident.

The PIV correlation data can also be used to determine integral scales, in the normal way. We define $R_{u u}$ as $R_{u u}(x+\Delta x, z+\Delta z)$, where $x, z$ is the reference location - the 'fixed probe' position and $\Delta x, \Delta z$ are spatial separations from that position. For $\Delta z=0$ we use $R_{u u}(\Delta x)$ or $R x_{u u}$; similar expressions hold for the vertical velocity correlations. Fig. 16a shows an example of an axial velocity spatial correlation for a point over the centre of a cube. These data correspond to a constant- $z$ slice (at $z / h=1.5$ ) through the contour plot in Fig. 15a and the figure includes similar correlation data obtained from the DNS computations of Coceal et al. (2006). These used a domain length of $16 \mathrm{~h}$ and the results agree well with the PIV data; shorter domain lengths, however, yielded significantly different behaviour, showing that a domain of at least $16 h$ is necessary for such data to be accurately obtained from simulations done using periodic boundary conditions. Note that one feature of the spatial correlations near the canopy top, which is certainly not typical of smooth-wall flows, is the clear evidence of a 'two-scale' behaviour. The data in Fig. 16a do not follow the commonly found exponential fall in $R_{u u}$ (away from $\Delta x=0$ ); rather, there is an initial fall close to an exponential $\left(e^{-\Delta x / L_{x}}\right)$ with a length scale of $L_{x} / h=1.5$, followed at larger separations by a fall that more closely follows $L_{x} / h=3.5$; these two trends are included in the figure. This behaviour gradually disappears with increasing $z$ and is evidence that the flow around the canopy top is dominated by the canopyproduced turbulence, itself dominated by the strong shear layer around $z=h$, where one expects typical scales to be $\mathrm{O}(h)$. This behaviour is even more noticeable at locations between the cubes, as illustrated in Fig.16b which shows additional data at $\mathrm{P} 1$ and $\mathrm{P} 2$, for $z / h=1.2$. The apparent differences 

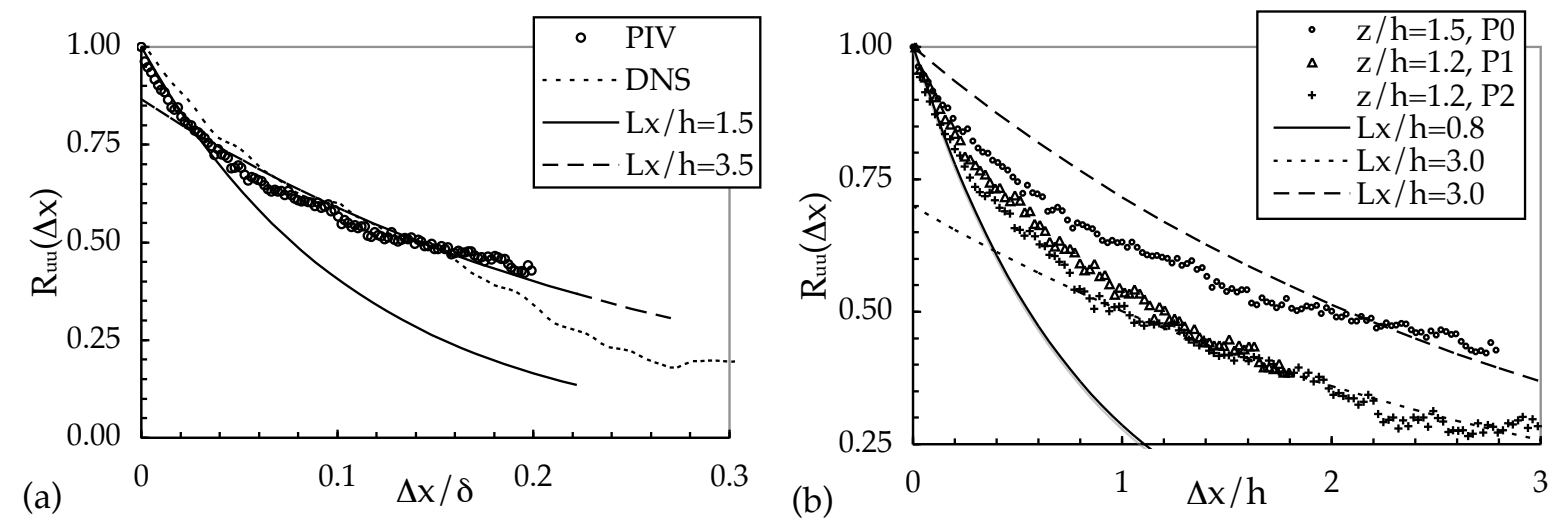

Fig. 16 Two-point axial velocity spatial correlation variation with axial separations. (a): $z / h=1.5$ over cube centre (P0). Solid and dashed lines are $R_{u u}=e^{-\Delta x / L_{x}}$ with $L_{x} / h=1.5$ and 3.5, respectively. (b): $z / h=1.5$, $\mathrm{P} 0 ; z / h=1.2$ for $\mathrm{P} 1$ and $\mathrm{P} 2$; lines refer to exponential variations with $L_{x} / h=0.8$ and 3.0. Note the different $R(0)$ values chosen for two of the exponential cases.

in scales suggested by the small and large separation behaviour of $R x_{u u}$ is almost a factor of four for P1. In the outer layer (data not shown), the scale is more closely linked to the boundary layer depth. $L_{x}=3.5 h$, for example, corresponds to $L_{x} / \delta=0.44$ - close to the 0.42 found by Ganapathisubramani et al. (2005) at $z / \delta=0.5$. Overall, detailed results indicate that $L x_{u u}$ initially increases with height before decreasing in the outer layer, a trend similar to that typically found in a smooth-wall layer (e.g., Ganapathisubramani et al., 2005).

Figs.17 and 18 present shaded contour plots of $R_{u u}$ and $R_{w w}$ for three vertical locations of the reference (unity correlation) point at the P1 and P2 positions. Recall that P1 (Fig.1) is just behind a cube whereas P2 is just ahead of one. What is most noticeable about the correlation behaviour as the reference point moves down into the canopy region is that the axial velocity correlations become relatively much more restricted in extent than do the vertical velocity correlations - compare (a-c) with (d-f) in both figures. It is clear too that this restriction is more significant at P1 than at P2 (compare, for example, Fig.17b with Fig.18b). On the other hand, the vertical extent of the vertical velocity correlations is much less affected at both locations.

These (and other similar) correlation data were used to deduce the length scales, $L x_{u u}, L z_{u u}$, $L x_{w w}$ and $L z_{w w}$ along the $\mathrm{P} 1$ and $\mathrm{P} 2$ lines. These scales are defined as the separations at which the appropriate spatial correlation has fallen to 0.5 - a common definition. (The available PIV domain size was sometimes too short to give correlations below about 0.4 , preventing use of a definition based on the separation at $R=0.368$, which for an exponential correlation would yield the scale, $L$, in $R=e^{-\Delta x / L}$.) The results are shown in Fig. 19. A logarithmic scale is used in Fig. 19a to clarify the behaviour for $z<h$. It is worth emphasising that if PIV were not used, data like these could only be obtained in these highly turbulent regions using two separate LDA systems. Notice first that at both 

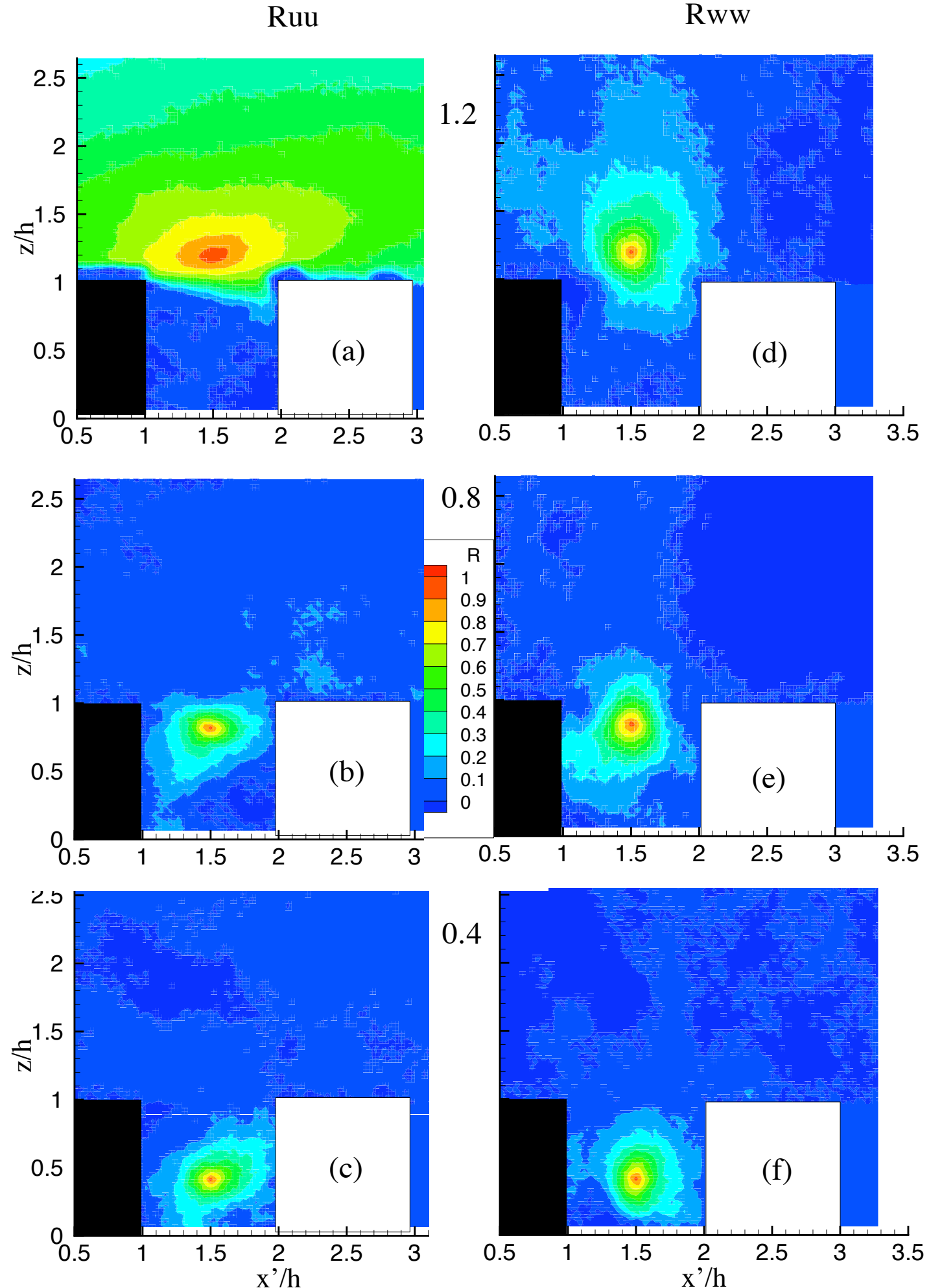

Fig. 17 Correlation contours for $R_{u u}(\mathrm{a}, \mathrm{b}, \mathrm{c})$ and $R_{w w}(\mathrm{~d}, \mathrm{e}, \mathrm{f})$ at $\mathrm{P} 1$. Numbers between the figures refer to the $z / h$ values of the correlation origin. $x^{\prime}=0$ is here defined as the upstream face of the cube cut by the light sheet. 


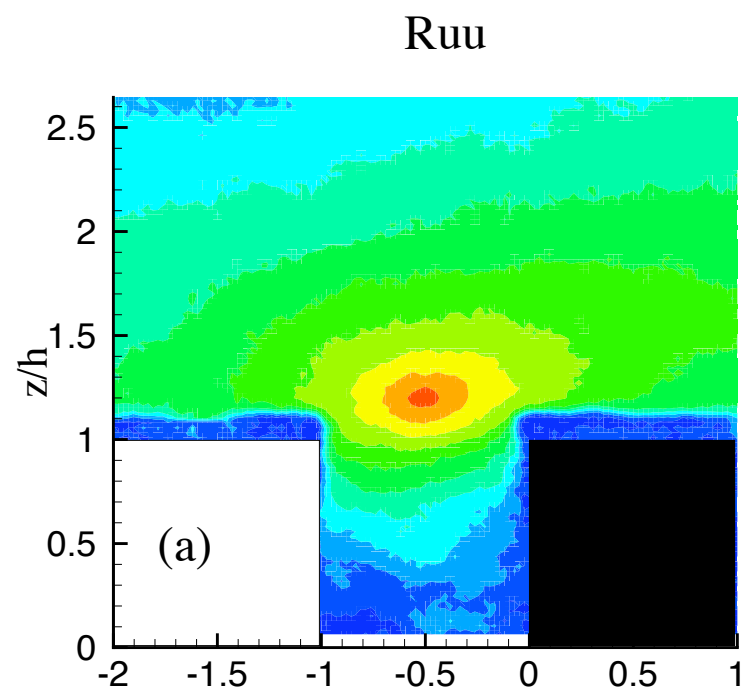

\section{Rww}
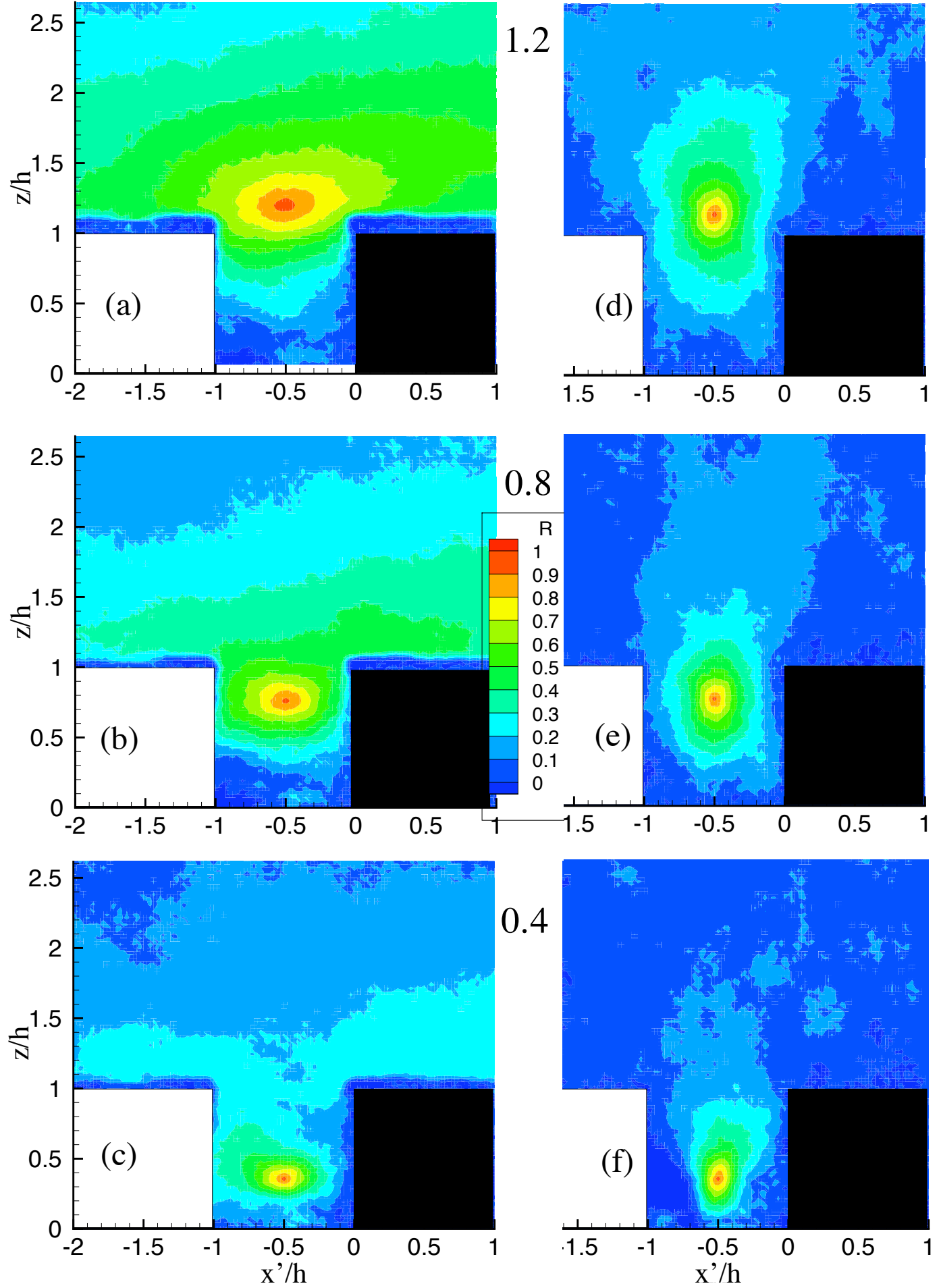

Fig. 18 Correlation contours for $R_{u u}(\mathrm{a}, \mathrm{b}, \mathrm{c})$ and $R_{w w}(\mathrm{~d}, \mathrm{e}, \mathrm{f})$ at $\mathrm{P} 2$. Numbers between the figures refer to the $z / h$ values of the correlation origin. $x^{\prime}=0$ is here defined as the upstream face of the cube cut by the light sheet. 

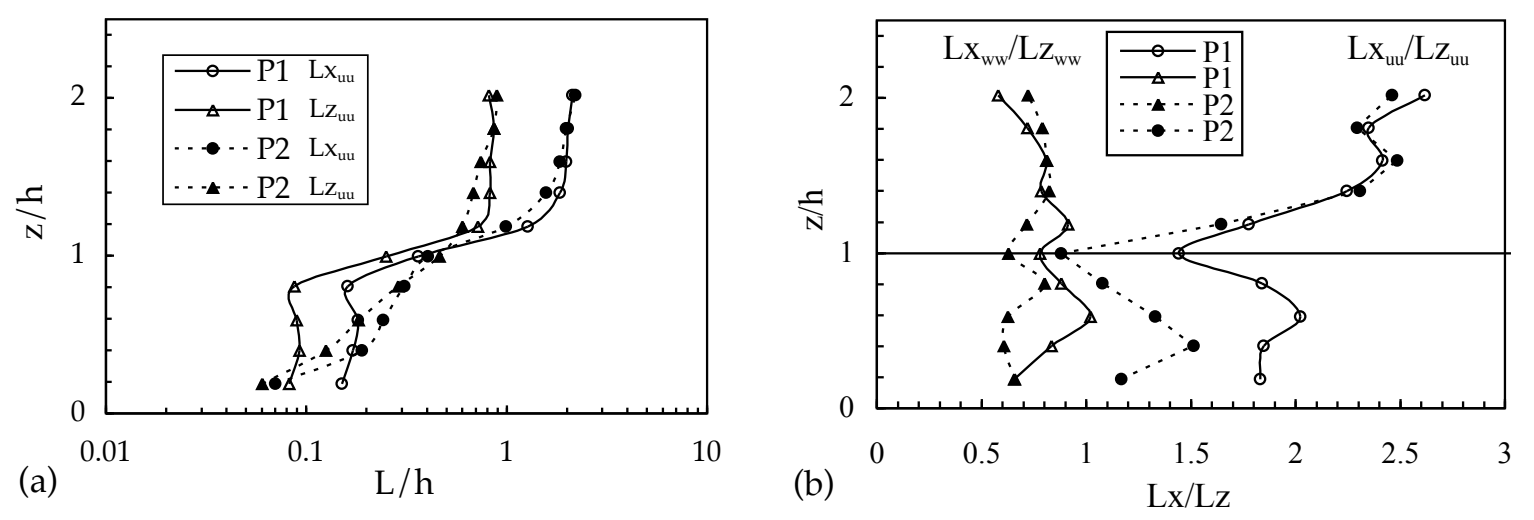

Fig. 19 (a): Length scales, $L x_{u u}$ and $L z_{u u}$, through the canopy region. Open and solid symbols are for P1 (behind the cube) and P2 (in front of the cube), respectively; circles refer to $L_{x}$ with horizontal 'probe' separations and triangles are for vertical separations. (b): Ratios of axial to vertical length scales for the axial and vertical velocity components.

locations the most rapid changes in scale occur around $z=h$, as suggested by the contour plots in Figs. 17 and 18. However, secondly, at P1 once $z<0.8 h$ the very small value of $L x_{u u}$ remains roughly constant. Recall that in this region the dominant mean flow direction is actually vertical (see Fig. 6a), so one should perhaps not interpret $L x_{u u}$ in the usual way. At P2 there is a much less abrupt variation in scales; this is not surprising, given that this location is some way downstream of the upstream cube ( $2.5 h$ from its downstream face) so the shear layer around $z=h$ is relatively thick. The mean flow direction is predominantly in the $x$-direction except for $z<0.4 h$. Below that height, the P2 (i.e. $x=-0.5 h)$ profile cuts the horseshoe vortex, see Fig. $6 \mathrm{~b}$, within which both length scales shown in Fig. 19a have very low values and are, in fact, very similar. Fig.19b shows the ratio of the length scales with vertical and axial separations, for both velocity components. Notice in particular that at both P1 and P2 the ratio lies within the range $0.5-2$ once $z / H$ falls below about 1.2 , which is indicative of much more isotropic (spherical) eddies than those that exist well above the canopy. In the latter region the data are more typical of those that occur over smooth walls, being characterised by much larger ratios of $L x_{u u} / L z_{u u}$ (exceeding about 2.5) and, simultaneously, very similar values of $L x_{w w}$ and $L z_{w w}$ which are both much smaller than the axial velocity length scales. As noted previously, the present data have features similar to those found in forest canopies. Katul \& Chang (1999), for example, deduced integral length scales within and just above a canopy (indirectly, since only single point data were obtained); their results are in some respects similar to those shown in Fig. 19. The similarities between flows in dense forest canopies and the present urban-type canopy is a noteworthy outcome of the present work.

These data confirm the earlier conclusions that within the canopy the turbulence characteristics are strongly dependent on spatial position and that near the surface the turbulence is more closely isotropic, with typical scales smaller, by an order of magnitude, than those in the roughness sublayer 
just above $z=h$. This is all indicative of a redistribution of turbulence energy between the three components, as has been suggested for the roughness sublayer region of other rough-wall geometries by previous authors (e.g. Krogstad et al., 2005, Lee \& Sung, 2007). Such redistribution is clearly even more complete within the canopy region. Note that these length scale data confirm that the roughness sublayer extends to around $z=2 h$; at that location (and above, not shown) the scales at P1 and P2 coincide (see Fig.19). They also agree closely with the scales obtained by CCR, much more laboriously, using simultaneous LDA and hot-wire systems to obtain the data in the restricted regions where the latter was viable. Finally, some comments about dispersive stresses within the canopy are appropriate. Cheng \& Castro (2002) showed that above the canopy region these stresses are negligible compared with the regular Reynolds stresses. However, neither in those experiments nor in the ones presented here were sufficient profiles obtained within the canopy to deduce the dispersive stresses. But recent Direct Numerical Simulations of Coceal et al. (2006) for the identical cube array as in the present experiments confirm that the dispersive stresses are indeed significant within the canopy. Similarly, Xie et al.'s (2008) Large Eddy Simulations of flow over an array of random height obstacles has a similar conclusion. In the context of plant canopies there is also some evidence that dispersive stresses can be significant (Bohm et al., 2000, Poggi et al., 2004a), particularly for relatively sparse element arrays. For urban canopies, it would be interesting to know how dense they must be before dispersive stresses become insignificant, as they seem to do for sufficiently dense plant canopies.

\section{Final discussion and conclusions}

We conclude that around the top of the canopy region the flow is very different from that in the nearwall region of smooth-wall boundary layers. Sweeps are dominant and provide a large contribution to the mean shear stress, particularly those characterised by the strongest motions. Ejections only become (relatively) significant outside the roughness sub-layer, which extends to about $z=2 h$, and then follow roughly what would be expected for a smooth surface. The sweeps must play a significant part in the behaviour of scalar pollutants which, if existing above the sub-layer will tend to be swept down into the street canyons. Pollutants arising deep within the canopy will be more strongly dispersed in the cross-wind directions within the canopy than able to escape into the flow aloft. This is not to say, of course, that local ejections of such pollutant into the flow above the canopy will never occur; but they are relatively rare events. This behaviour is crucially dependent on the roughness geometry. The present case of a staggered array with $25 \%$ area coverage is verging on what is often called 'skimming' flow; more open arrays tend to be better ventilated (see Kastner-Klein et al., 2004, for a discussion). This implies that the turbulence structure of the canopy flow must also be quite dependent on the details of the geometry, which is well illustrated by the numerical computations of Coceal et al. (2006, 2007) who show how different the canopy flow is if the cubes are arranged in a square, rather than a 
staggered pattern. In particular, both Coceal et al. (2007) and Xie et al. (2008) argue convincingly on the basis of detailed numerical simulation data that it is difficult to characterise even the mean canopy flow in a completely general way; this is not, perhaps, surprising. The present results should not therefore be taken as being indicative of what would necessarily occur for other roughness patterns, or even other wind directions. Nonetheless, the fact that turbulent energy redistribution, from vertical to spanwise components, occurs within the roughness sub-layer, thus decreasing anisotropy levels and, in the canopy, strongly reducing typical turbulent scales, seems to be a common feature of very rough-wall flows, including those over dense vegetation canopies.

Acknowledgements This work could not have been undertaken without the willing help of technical staff in the School of Engineering Sciences; in particular we are indebted to Mr M Tudor-Pole and the late Dr C Williams. RR thanks the School for a Research Scholarship. The work was supported by the UWERN programme of the Natural Environment Research Council (under NCAS grant number DST/26/39), for which we are very grateful. We also wish to thank the anonymous referees for a number of helpful comments.

\section{References}

Adrian R, Meinhart C, Tomkins C (2000) Vortex organisation in the outer region of the turbulent boundary layer. J. Fluid Mech. 422: 1-54

Bottema M (1997) Urban roughness modelling in relation to pollution dispersion. Atmos. Environ. 31: 3059-3075.

Castro IP (2007) Rough wall boundary layers: mean flow universality. J. Fluid Mech. 585: 469-485

Castro IP, Cheng H, Reynolds R (2006) Turbulence over urban-type roughness: deductions from wind-tunnel measurements. Bound. Layer Meteorol. 118: 109-131

Castro IP, Robins AG (1977) The flow around a surface-mounted cube in laminar and turbulent streams. J. Fluid Mech. 79:307-335

Cheng H, Castro IP (2002) Near-wall flow over urban-like roughness. Bound. Layer Meteorol. 105: 411-432

Cionco RM (1972) A wind profile index for canopy flow. Bound. Layer Meteorol. 3: 255-263

Coceal O, Belcher SE (2004) A canopy model of mean winds through urban areas. Q.J.R. Met. Soc. 130: $1349-1372$

Coceal O, Thomas TG, Castro IP, Belcher, SE (2006) Mean flow and turbulence statistics over groups of urban-like cubical obstacles. Bound. Layer Met. 121: 491-519

Coceal O, Thomas TG, Belcher, SE (2007) Spatial variability of flow statistics within regular building arrays. Bound. Layer Met. 125: 537-552

Davidson MJ, Snyder WH, Lawson RE, Hunt JCR (1996) Wind tunnel simulations of plume dispersion through groups of obstacles. Atmos. Environ. 22: 3175-3731

DeGraaff D, Eaton J (2000) Reynolds-number scaling of the flat-plate turbulent boundary layer. J. Fluid Mech. 422: 319-346

Erm L, Joubert P (1991) Low Reynolds number turbulent boundary layers. J. Fluid Mech. 230: 1-44

Finnigan J (2000) Turbulence in plant canopies. Ann. Rev. Fluid Mech. 32: 519-572

Ganapathisubramani B, Hutchins N, Hambleton W, Longmire E, Marusic I (2005) Investigation of large-scale coherence in a turbulent boundary layer using two-point correlations. J. Fluid Mech. 524: 57-80

Grachev Y, Hunt JCR (2006) (Eds.) Flow through plant canopies and urban terrains. NATO-ASI Summer School, Kluwer. 
Grimmond CSB, Oke TR (1999) Aerodynamic properties of urban areas derived from analysis of surface form. J. Appl. Meteorol. 38: 1262-1292

Hunt JCR, Eames I, Westerweel J (2006) Mechanics of inhomogeneous turbulence and interfacial layers. J. Fluid Mech. 554: 499-519

Jiménez J (2004) Turbulent flows over rough walls. Ann. Rev. Fluid Mech. 36: 173-196.

Kastner-Klein P, Rotach M (2004) Mean flow and turbulence characteristics in an urban roughness sublayer. Bound. Layer Meteorol. 111:55-84

Kastner-Klein P, Berkowicz R, Britter RE (2004) The influence of street architecture on flow and dispersion in street canyons. Meteorol. Atmos. Phys. 87: 121-131

Katul GG, Chang WH (1999) Principal length scales in second-order closure models for canopy turbulence. J. Appl. Meteorol. 38: 1631-1643

Krogstad PA, Antonia RA, Browne L (1992) Comparison between rough- and smooth-wall turbulent boundary layers. J. Fluid Mech. 245: 599-617

Krogstad PA, Antonia RA (1999) Surface roughness effects in turbulent boundary layers. Expts. in Fluids 27: 450-460

Krogstad PA, Andersson, HI, Bakken OM, Ashrafian A (2005) An experimental and numerical study of channel flow with rough walls. J. Fluid Mech. 530: 327-352

Lee S-H, Sung HJ (2007) Direct numerical simulation of the turbulent boundary layer with a rod-roughened wall. J. Fluid Mech. 584:125-146

Lu S, Willmarth W (1973) Measurements of the structure of the Reynolds stress in a turbulent boundary layer. J. Fluid Mech. 60: 481-511

MacDonald R (2000) Modelling the mean velocity profile in the urban canopy layer. Bound. Layer Meteorol. 97:25-45

Martinuzzi RJ, Tropea C (1993) The flow around surface-mounted prismatic obstacles placed in a fully developed channel flow. ASME J. Fluids Eng. 115: 85-92

Marusic I (2001) On the role of large-scale structures in wall turbulence. Phys. of Fluids 13: 735-743

Poggi D, Katul GG, Albertosn JD (2004a) A note on the contribution of dispersive fluxes to momentum transfer within canopies. Bound. Layer Meteorol. 111: 615-621

Poggi D, Porporato A, Ridolfi L, Albertosn JD, Katul GG (2004b) The effect of vegetation density on canopy sub-layer turbulence. Bound. Layer Meteorol. 111: 565-587

Raupach M (1981) Conditional statistics of Reynolds stress in rough-wall and smooth-wall turbulent boundary layers. J. Fluid Mech. 108: 363-382

Raupach M, Antonia RA, Rajagopalan S (1991) Rough-wall turbulent boundary layers. Appl. Mech. Rev. 44: $1-25$

Reynolds RT, Hayden P, Robins AG, Castro IP (2007) Spanwise variations in nominally two-dimensional rough-wall boundary layers. Expts. In Fluids. 42, 311-320

Rotach MWL, Vogt R, Bernhofer C, Batchvarova E et al. (2007) BUBBLE - An urban boundary layer meteorology project. Theoret. \& Appl. Climat. 81: 231-236.

Tutu N, Chevray R (1975) Cross wire anemometry in high intensity turbulence. J. Fluid Mech. 71: 785-800

Xie ZT, Coceal O, Castro IP (2008) Large-Eddy Simulation of flows over random urban-like obstacles.

Bound. Layer Meteorol. (in press) 